Verdünnen von $26,3 \mathrm{~cm} 1 / 10 \mathrm{~N}$.- Natriumthiosulfatlösung auf 1 Liter erhalten wird; von dieser Lösung zeigt jedes verbrauchte cem $0,1 \mathrm{mg}$ oder, auf $1000 \mathrm{ccm}$ Wasser bezogen, $1 \mathrm{mg} \mathrm{N}_{2} \mathrm{O}_{3}$ an. - Folgende Bestimmungen beweisen die Genauigkeit des Verfahrens :

\begin{tabular}{|c|c|c|c|c|c|c|c|c|c|c|c|}
\hline \multicolumn{12}{|c|}{$\mathrm{N}_{2} \mathrm{O}_{3}$ in $1000 \mathrm{ccm}$ destilliertem Wasser } \\
\hline $\mathrm{ngew}$ & endet & gefun & den & an $g \in w$ & endet & $g$ efur & aden & angew & endet & $g \in f u r$ & den \\
\hline 5,00 & $\mathrm{mg}$ & 4,99 & $\mathrm{mg}$ & 2,50 & $\mathrm{mg}$ & 2,42 & $\mathrm{mg}$ & 0,50 & $\mathrm{mg}$ & 0,50 & $\mathrm{mg}$ \\
\hline 5,00 & $\eta$ & 4,98 & $n$ & 1,00 & 6 & 0,97 & 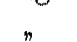 & 0,50 & $\pi$ & 0,49 & , \\
\hline 5,00 & 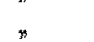 & 4,90 & * & 1,00 & 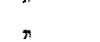 & 0,96 & " & 0,25 & $n$ & 0,26 & : \\
\hline 2,50 & 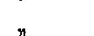 & 2,50 & 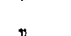 & 1,00 & . & 0,92 & 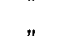 & 0,25 & , & 0,24 & 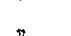 \\
\hline 2,50 & $"$ & 2,40 & " & 0,50 & $"$ & 0,50 & , & 0,25 & $"$ & 0,24 & n \\
\hline
\end{tabular}

Vorbandenes Ferrieisen stört nicht, wenn man, wie jetzt vorgeschrieben, zum Ansäuern Phosphorsäure verwendet. Dagegen wirken sowohl Ferroeisen als organische Stoffe natürlich in viel höherem Maße störend als bei der Zeitmethode, da ja die Menge des zur Ausscheidung gelangenden Jods bei dieser Methode gering ist. Das Ferroeisen entfernt man in derselben Weise, wie dies bei der Zeitmethode angegeben wurde; auch die Bestimmung des durch reduzierende Substanzen verursachten Fehlers wird in schon angegebener Weise bewerkstelligt.

Zusammenfassend ergibt sich aus vorliegenden Untersuchungen, daß zur Bestimmung der salpetrigen Säure in damit verunreinigten Wässern die jodometrischen Methoden besonders geeignet sind, und zwar wird man, je nachdem die salpetrige Säure in geringerer oder bedeutenderer Menge vorhanden ist, entweder die Zeitmethode oder die Bicarbonatmethode benutzen.

Herr Dr. A. Loczka war mir auch bei diesen Untersuchungen mit großem Fleibe behilflich, wofür ich ihm freundscháftlich danke.

\title{
Über die Samen von Chenopodinm album L.
}

\author{
Von \\ Regierungsrat Dr. T. F. Hanausek. \\ [Eingegangen an 14. November 1914.]
}

Vorkommen im Getreidemeble. Mitunter findet man in gröberen Mehlen, insbesondere in Roggenmehlen, unter den von Unkrautsamen herrührenden Beimengungen sehr kleine braune und opake schwarze Partikel, von denen die helleren durchscheinenden bei flüchtiger Betrachtung als Bestandteile von Wickensamenschalen angesehen werden können und auch wahrscheinlich oft angesehen worden sind. Die schwarzen Partikel sind so, wie sie sich ohne weitere Behandlung präsentieren, überhaupt nicht zu bestimmen, höchstens könnte man sie für Kohlenteilchen halten. Erst nach Auf- 
hellung in J avelle'scher Lauge oder in anderen Aufklärungsmitteln lassen sie eine Struktur erkennen, die mit der der braunen Partikel identisch ist. Bei eingehender Beobachtung wird man aber sofort wahrnebmen, daB ihr Bau von dem der Wickensamenschalen gänzlich verschieden ist, wie unten des nähern dargetan wird. Sie gehören den Samenschalen verschiedener M el den-Arten an, und zwar aus den Gattungen A triplex und Chenopodium, die gemeine Unkräuter des bebauten Landes sind und micht selten die Randstellen der Getreidefelder in größerer Menge besiedeln. Hauptsächlich ist es das vielgestaltige Chenopodium album L., der gemeine GänsefuB - wegen der abführenden Wirkung des Krautes auch Schießmelde, in Tirol nach M urr Scheißmalte genannt -, dessen Samen am häufigsten (neben Chenopodium murale L.) im Mehle anzutreffen sind. Das Vorkommem desselben im Mehle mikroskopisch nachweisen zu können, gab Veranlassung zu vorstehender Mitteilung.

Chenopodiumsamen als Brotfrucht, Russisches Hungerbrot. Aber auch noch andere Gründe lassen diese Mitteilung als nicht unzeitgemäß erscheinen. Seit uralter Zeit wird in Peru und Chile die Quinoa, das Che nopodium Quinoa L., unserem Chenopodium abum sehr nahestehend und sehr ähnlich, als Brotfrucht angebaut und aus den Samen ein Brotmehl dargestellt. Nebenbei bemerkt ist die Quinoa im Mittelmeergebiete, in neuerer Zeit auch in Deutschland, der Schweiz und. in Frankreich als Futterpflanze in Kultur. Von Chenopodium album ist bekannt, daß es schon in vorgeschichtlicher Zeit ${ }^{1}$ ) eine Kulturpflanze war; außer den mehlreichen Samen wurden wahrscheinlich auch die jungen Sprosse als Gemüse verwendet, wie letzteres auch heute noch in manchen Gegenden der Fall sein soll. Ich möchte annehmen, daß die Erinnerung an diese prähistorische Verwendung der Samen als Brotfrucht nicht erloschen ist, denn die Verwendung taucht immer wieder auf, wenn Mißernten eine Hungersnot zur Folge haben. Sehr bekannt ist die Verwendung der Samen in Rubland in den Jahren 1891-1892, wo das Chenopodiummebl in Vermischung mit Roggenmehl zu dem sogenannten russischen Hungerbrot verbacken wurde. Einer meiner Schüler, Herr P. Kentmann, ein Russe, sandte mir 1891 ein Muster dieses Brotes, das ich jetzt wieder einer näheren Untersuchung unterwarf.

Neuesten Zeitungsnachrichten zufolge (Münchner Neueste Nachrichten vom 5. November 1914) ist das russische Hungerbrot mit Chenopodiammehl wieder aufgetaucht, und zwar soll letzteres, von den Samen von Chenopodium murale L., dem Mauer-Gänsefuß, stammen.

Brot und Mehl sind damals (1892-93) von verschiedenen deutschen Forschern eingehenden Untersuchungen unterzogen worden, aus denen hervorgeht, daß das Chenopodiummehl, wie es im Hungerbrot vorkommt und vermutlich nur recht primitiv gewonnen wird, als ein nur wenig geeignetes Ersatzmittel des Getreidemehls ${ }^{2}$ ) angesehen

1) Buschau, Vorgesch. Botanil, S. 150; nach A scherson-Graebuer, Synopsis der mitteleurop. Flora, V, S. 39.

2) Th. W a a ge hat in einem Artikel über "Getreidebrot und seine Ersatzmittel" (Pharmaz. Zentralhalle 1891, No. 48) eine Liste der Vegetabilien zusammengestellt, die zu Brotmehl dienen könnten und gedient haben (auch Chenopodium ist darin enthalten) und hebt nach $M$ urray (Apparatus medicaminum 1788) Roß̉kastanien (l. c. IV, S. 49), Eicheln (1. c, I, S. 50) und Lupinen (l. c. II, S. 288) hervor. Die Schlüsse seiner Ausfübrungen, die auch für die gegenwärtige Kriegszeit Bedentung haben mögen, besagen, dass "1. die Verwenđung entbitterter 
werden kann, auch wenn man von dem sehr geringen Ertrag absieht, der durch die Kleinheit der Samen bedingt ist.

Von diesen Arbeiten sei zunächst die von Virchow und Salkowski ${ }^{1}$ ) angeführt, der zufolge russisches Hungerbrot in den Distrikten an der Wolga aus den Samen von Chenopodium murale erzeugt wurde. Nach der von Salkowski ausgeführten Analyse handelte es sich bei dem Hungerbrot um eine an Eiweiß und Fett sehr reiche Substanz, die, theoretisch betrachtet, einen ungewöhnlichen Nährwert besitzt und in dieser Beziehung sich an die sogenannten Proteinmehle anschliebt. Aus der nachstehenden Tabelle ist die chemische Zusammensetzung zu ersehen:

$\begin{array}{lcc} & \text { In der } & \text { In der } \\ \text { Wasser } & \text { natürlichen Substanz } & \text { Trockensubstanz } \\ \text { Eiweik } & 9,76 \% & - \\ \text { Fett } & 11,79 \% & 13,07 \% \\ \text { Stärke } & 3,79 \% & 4,20 \% \\ \text { Cellulose } & 36,52 \% & 40,47 \% \\ \text { Asche } & 15,06 \% & 16,69 \% \\ & 23,08 \% & 25,57 \%\end{array}$

Hierzu bemerkt der Referent der Vierteljabrsschrift, A. Halenke, daß es zweifelhaft erscheint, ob viel von den großen Mengen Eiweiß verdaulich ist. Auch die außerordentlich große Aschenmenge ist bezïglich ihrer Herkunft nicht aufgeklärt.

Ich möchte die Vermutung aussprechen, daß ein großer Teil der Asche nicht ein integrierender Teil des Samens ist, sondern von Erde herrührt, deren Beimengung bei der Ernte mit Hinsicht auf die außerordentliche Kleinheit der Samen sehr begünstigt wird. Was die Verdaulichkeit des Eiweißes betrifft, so könnte auf die Quinoa hingewiesen werden, die ein sehr altes Nahrungsmittel ist und dieses gewiß nicht hätte sein können, wenn es nur geringe Verdaulichkeit besäße; die Quinoa ist aber mit Ch. album so nahe verwandt, daß sie von Ascherson und Graebner2) unter die "Gesamtart" Ch. album mit einbezogen wurde. Die unten angegebenen Fütteruugsversuche sprechen freilich gegen eine ausgiebige Verdaulichkeit.

Nach dem russischen Botaniker Gordiag in (s. unten) ist Chenopodium album und nicht Ch. murale die Stammpflanze, deren Samen unter dem Namen Ljebeda zu Brotmehl verwendet werden.

Nach einer Analyse von G, Baumert und K. Halpern ${ }^{3}$ ) sind im russischen Hungerbrot enthalten:

Rokkastanien, Eicheln und Lupinen zur Brotbereitung sehr wohl möglich und anch bereits seit langer Zeit bekannt ist, daß aber 2. diese Verwendung nur unter ganz außergewöhnlichen Umständen, sicherlich aber nicht schon in gegenwärtiger Zeit empfehlenswert erscheint, daki vielmebr 3. einem wirklichen Mangel an Brotgetreide in den hauptsächlich Roggenbrot konsumierenden Staaten ungleich zweckmäßiger darch Einführung eines mit Roggenmehl versetzten Maisbrotes begegnet werden kann."

In einer Anmerkung weist der Verf. auf den Buchweizen hin, da dieser ohne weitere Behandlung verwendbar ist und Buchweizenmehl ein wohlschmeckendes Gebäck gibt.

Es ist gewif interessant, daf diese vor 23 Jahren geschriebenen Ausfuhrungen Wa a ge's für die Gegenwart zutreffen!

1) Vierteljahrsschr. f. Chemie d. Nahrungs- u. Genuß3m. 1893, 8, 54; nach Chem. Zentral. blatt $1893, \mathrm{I}, 168$.

$\left.{ }^{2}\right)$ L. c. V, S. 61.

3) Arehiv f. Pharm. 1898, 281, 644. 


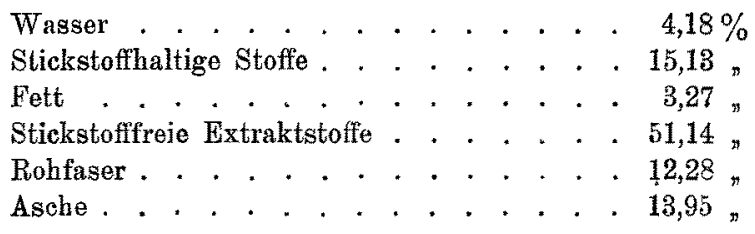

Auch aus dieser Analyse ergibt sich, daß der hohe Gehalt an Rohfaser und Asche den hohen Prozentsatz an stickstoffbaltigen Bestandteilen nahezu wertlos machen müßte. Nach einer Mitteilung der genannten Autoren wurden von Erismann und Salmenew Fütterungsversuche an Mäusen und Ratten angestellt, die ungünstige Resultate ergaben.

Schließlich soll noch erwähnt werden, daß Baumert und Halpern (l. c. 8. 648) aus den Samen von Ch. album Betain (Oxyneurin, $\mathrm{C}_{5} \mathrm{H}_{11} \mathrm{O}_{2} \mathrm{~N} . \mathrm{H}_{2} \mathrm{O}$ ) isoliert haben, das, wie es scheint, in Chenopodiaceen ziemlich verbreitet ist.

Bei der Verwendung des Samens zu Mehl darf auch die Beschaffenheit der Samenschale nicht außer acht gelassen werden; es mul untersucht werden, ob sie nicht durch größere Anhäufung in den Verdauungsorganen Reizungen der Schleimhäute - in Anbetracht der überaus starken Verdickung der Außenwände der Schalenepidermis und deren grofien Festigkeit - hervorruft und ob die kleinen Schalenteilehen zum größeren Teile in die Kleie abgeschieden werden können. In dieser Beziehung läbt sich aber auch wieder auf die schon so lange im Gebrauch stehende Quinoa hinweisen.

Morphologie und Anatomie des Samens. Der Samen von Chenopodium album hat einen kreisförmigen, mitunter sich nierenförmig verbreiternden Umriß und ist seitlich linsenförmig zusammengedrückt, er gleicht also in vollkommen ausgereiften Zustande einer Linse, deren Durchmesser 1 bis höchstens $1,5 \mathrm{~mm}$ beträgt, während die Dicke in der Linsenmitte $0,4-0,5 \mathrm{~mm}$ mißt. An der Stelle, die der Lage des Würzelchens entspricht, befindet sich ein mehr oder weniger deutlicher Vorsprung, der sich mitunter zu einer schnabelartigen Hervorragung vergrößert; einwärts desselben zeigt sich eine Vertiefung. Die Oberfläche ist tiefschwarz bis braun, glatt, wie lackiert glänzend und ohne jede Skulptur. Denn die oberflächliche Zeichnung, die man schon mit einer scharfen Lupe wahrnehmen kann, hat mit Hervorragungen bezw. Vertiefungen nichts zu tun. Dadurch unterscheidet sich dieser Samen schon auffällig von dem von $\mathrm{Ch}$. murale, da dieser eine fein-netzig-grubige, gänzlich glanzlose, also matte Oberfläche besitzt; auch ist er am Linsenrande etwas gekieltkantig, während der von Ch. album abgerundet erscheint. An nicht vollreifen Samen verläuft längs des Rückens, d. h. des Linsenrandes, eine Leiste, die an dem schnabelartigen. Vorsprunge endet und den Eindruck einer Schneckenwindung macht; sie entspricht dem stark vortretenden Embryo. Der Nabel ist nur sehr undeutlich auf der Samenbasis zu sehen, mitunter haftet noch als Rest des Funiculus ein faseriges lichtbräunliches Schöpfehen daran.

Aus der winzigen Größe des Samens ergibt sich schon eine äußerst geringe Ertragsfähigkeit mit Rüeksicht auf die Erntearbeit und eine gewisse Schwierigkeit, das Mehl von der Samenschale zu befreien.

An einem Flächenschnitt parallel zum Linsenrande (Fig. 1) gewahrt man unmittelbar unter der Samenschale den ringförmig verlaufenden Embryo, der das mehlweiße Nährgewebe umschließt (Fig. 1 pe). Der Scheitel des Würzelchens (Radi- 
cula, Fig. 1 rad) ist in dem schnabelartigen Vorsprunge am Samen auBen sichtbar. Die Keimblätter (Fig. 1 kot) sind ziemlich lang und schmalplankonvex. Von dem Nährgewebe, das als Peri sperm anzusprechen ist - das Endosperm ist im reifen Samen kaum mehr in Resten wahrnehmbar - hebt sich der Keim durch sein gelbliches, durchscheinendes Aussehen deutlich ab. In Fig. 2 ist ein Samenquerschnitt (in der Richtung der Linie $\mathbf{x}-\mathbf{x}$ bei Fig. 1) in 60-facher Vergrößerung von einem noch nicht vollreifen Samen gezeichnet, der daher noch das scharfe Abgesetztsein der Radicula und die starke Rundung des Rückens bei den Kotyledonen zeigt. Man sieht, daß das Perisperm und die plankonvexen Keimblätter unmittelbar innerhalb der zweischichtigen Samenschale liegen, während zwischen der stilrunden Radicula (Fig. $2 \mathrm{rad)}$ und der Samenschale (Fig. 2, 1,2) noch eine Schicht (4) eingeschaltet ist, die wahrscheinlich einen Rest des Endosperms darstellt. Weil der Samen noch

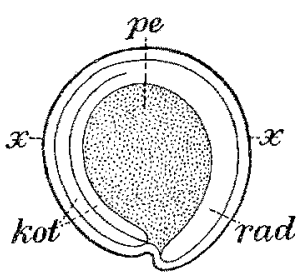

Fig. 1.

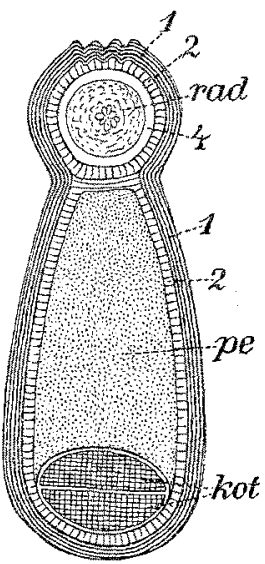

Fig. 2.

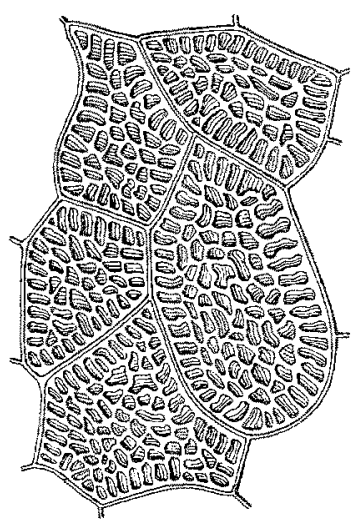

Fig. 3.

nicht vollreif war, zeigt auch sein Querschnitt noch nicht die linsenförmige Kontur. Auf der Radienlarseite verläuft der Umriß nicht im Bogen, sondern wellenförmig, daber besitzt die Samenschale daselbst vertiefte Streifen oder' Furchen.

Die Epidermis der Samenschale ist durch einen sehr eigentümlichen Bau ausgezeichnet, der schon öfters Gegenstand der Untersuchung gewesen ist. Arbeiten über die Anatomie der Chenopodiumsamen liegen von Marloth, A. M e u n i e r ${ }^{1}$ ) und A. Gordiagin ${ }^{2}$ ) vor; die des letzteren befaßt sich speziell mit Ch. album und bietet eine eingehende und sehr genaue Darstellung, soweit dies aus dem von dem Verf. selbst herrührenden Referat im Botan. Zentralblatt ersehen werden kann; die Originalarbeit ist mir nicht zugänglich.

Die Epidermis setzt sich aus in der Fläche polygonalen Zellen zusammen, deren Wände aber häufig gekrümmt sind (Fig. 3); nur am Rücken, dem Sameurande, sind sie rechteckig oder quadratisch und in $3-4$ gleichlaufenden Reihen geordnet,

1) A. Meunier, Les téguments seminanx des Cyclospermes, Partie I, La Cellule, T. VI, Fasc. 2. - Zit, nach Uhlworm, Bot. Zentralbl. 1892, ̌1, 59.

2) A. Gordiagin, Tageblatt der Gesellschaft der Arzte zu Kasan 1892. Lief. 2. Nach des Verf's. Ref. im Bot. Zentralbl. 1893, ǒ5, 163. 
An der Außenfläche zeigt jede Zelle überaus zahlreiche, nahe aneinander anschließende, verschieden und unregelmäßig begrenzte, durch dunkle Färbung kenntliche Stellen, von denen die an den Zellrändern liegenden gestreckt, parallel zueinander und senkrecht zur Zellgrenzlinie gestellt sind, was den Zellrändern ein kurz-grobstreifiges Aussehen verleiht. Diese Stellen sind, wie schon bemerkt, keine Hervorragungen, sondern die Basen eigentümlicher Kutikularbildungen, worüber der Querschnitt (Fig. 4 u. 5) Aufschluß gibt. Da zeigt sich nun, daß die Außenwand der Epidermiszelle außerordentlich mächtig verdickt ist, während die Seiten- und Innenwände nur sehr dünn, erstere oft zusammengedrückt und verkürzt sind, wodurch das Lumen stark an Raum verliert. Wie sehr die Räumlichkeit des Lumens verschieden sein kann, zeigen die beiden Figuren 4 und 5, 1. Die dicke Außenwand ist nicht homogen, sondern zeigt in einer gleichförmigen Grundmasse eingebettet schmale, sehr spitze, quergestreifte, kegelförmige Zapfen, deren Basen die an der Aubenseite der Epidermiszellen sicht-

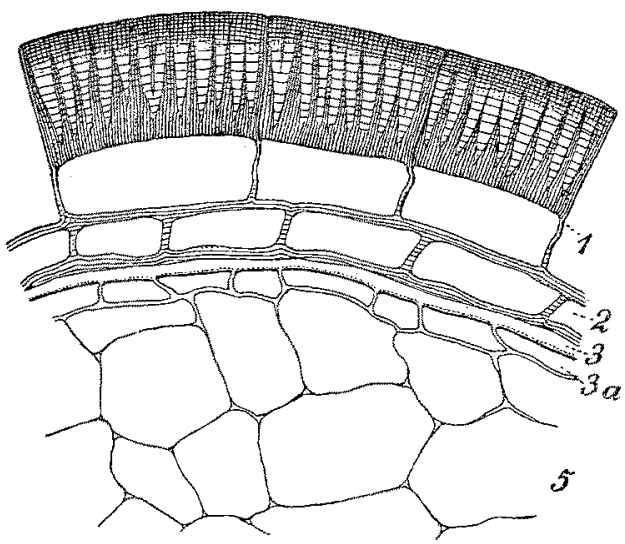

Fig. 4.

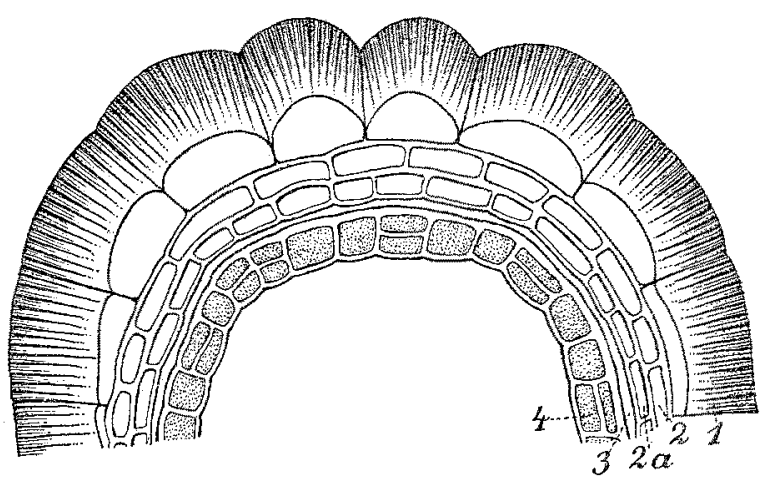

Fig. 5.

baren dunkelgefärbten Stellen sind und deren Spitzen der Innenseite der Außenwand, also dem oberen Lumenrande zustreben, aber ihn nicht erreichen. Meunier hat diese Zapfen in zutreffender Weise mit Stalaktiten verglichen. Thre Zahl ist in jeder Zelle sehr bedeutend; sie heben sich durch ihre lichtere Färbung von der homogenen Verdickungsmasse deutlich ab. Ist die Samenschale tiefschwarz, so ist auch am Querschnitt die obere Hälfte der Auf̧enwand infolge der starken Infiltration durch den Farbstoff dunkel und undeutlich; erst durch Kochen in Kalilauge, wodurch der Farbstoff blaugrün gelöst wird, oder durch längere Einwirkung von Javelle'scher Lauge werden die basalen Partien der Zapfen sichtbar. Nach Meunier ist dieses Vorkommen ein charakteristisches Merkmal der (yclospermen ${ }^{1}$ ).

Nach ihrem mikrochemischen Verhalten sind diese Zapfen Kutikularbildungen. Gordiagin sagt darüber folgendes: „Die in den Zwischenräumen der Kegel befindliche Grundmasse der Zellhaut ist weniger kutikularisiert, da sie sich mit Jod und Schwefelsäure (in unreifen Samen) schmutzigblau färbt, während die Kegel unverändert bleiben. Behandelt man auf dem Objektträger einen Querschnitt aus der

$\left.{ }^{1}\right)$ Auch bei den Caryophyllaceen ist es gefunden und zuerst von Hegelmaier beschrieben worden. 
Samenschale mit dem Schultze'schen Gemische unter Erhitzung und kocht darauf vorsichtig in Kalilauge, so verschwinden die pigmentierten Kegel gänzlich und die Wand wird homogen. Nach dieser Behandlung wird die gesamte obere Wand von Chlorzinkjodlösung blau gefärbt, jedoch färben sich diejenigen Stellen, wo die oben erwähnten Kegel (Differenzierungsstäbchen im Sinne Hegelmaier's) sich befanden, schwächer als die zwischenliegende Grundmasse. Die schwach pigmentierte bezw. im unreifen Samen ganz ungefärbte Grundmasse kann bei Flächenansicht den Anschein von Poren hervorrufen."

In vielen Präparaten fand ich das Lumen der Epidermiszellen leer, hier und da enthalten diese Zellen und die der folgenden Schicht einen braunen homogenen Inhaltskörper, der im Aussehen an die sogenannten Inklusen erinnert, aber keine Gerbstoffreaktion gibt und sich auch sonst ganz anders verhält. Er dürfte ein Pigment darstellen.

Unter der Epidermis liegt ein Gewebe, das bei reifen Samen in der vom Perisperm eingenommenen Abteilung (Fig. 4, 2) aus einer, in der Radikular-Abteilung aus zwei und auch aus mehreren Zellreihen besteht (Fig. 5, 2, 2a). An der Grenze

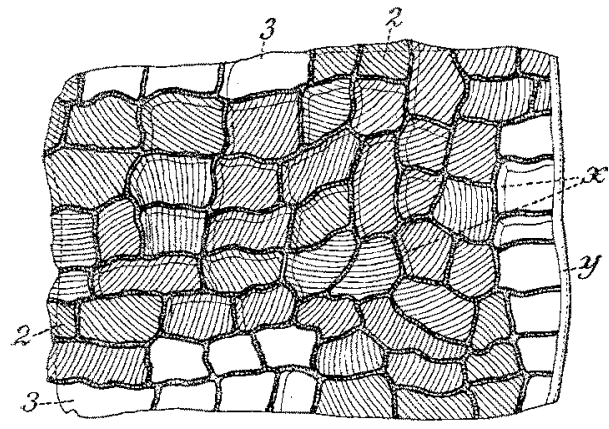

Fig. 6.

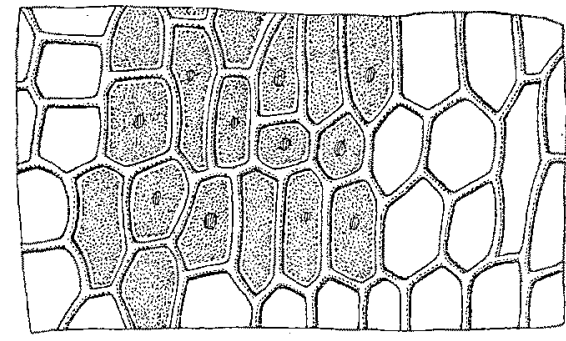

Fig. 7.

zwischen Perisperm- und Radikularteil (Fig. 2) teilt es sich und schließt die beiden Teile voneinander $a b$. Dieses Gewebe ist bräunlich, wird durch Jod und Schwefelsäure und durch Chlorzinkjod nur dunkelbraun gefärbt und besteht demnach nicht aus Cellulose; auch Phloroglucin-Salzsäure bewirkt keine auffällige Farbveränderung; man muß demnach annehmen, dah das Gewebe cutinisiert ist, eine Art Kork darstellt. In der Flächenansicht erscheinen die Zellen unregelmäßig-vierseitig (Fig. 6), ibre Außenfläche, mit dex sie an die Epidermis grenzen, ist fein aber scharf gestreift, die Orientierung der Streifenlagen ist für einzelne Zellen oder Zellgruppen verschieden. Die Zellwände sind nicht, weder in der Flächenansicht, noch im Querschnitt, gleichmäbig stark, d. h. sie sind nicht eben und parallellinig begrenzt, sondem bald dicker bald dünner, wie dies aus den Zeichnungen 4 und 6,2 deutlich ersehen werden kann. Die an der Außenfläche liegenden Streifen sind die Falten der auflagernden Cuticula. An einem Flächenpräparat sieht man sehr häufig neben und unter der braunen Wand der Zellen noch eine zweite anscheinend farblose Wand durchschimmern (Fig. 6 bei $\mathrm{x}$ ). Der Querschnitt zeigt, daß diesem Gewebe ein farbloser, 2,5-3,4 $\mu$ dicker, durchweg gleichstarker, hy aliner, anscheinend strukturloser Streifen (Fig. 4, 3) anliegt, dem vielleicht auch die vorbin genannten farblosen Wände angehören. Er gibt gleichfalls keine Cellulose-Reaktion und ist so fest mit der braunen 
Schicht verbunden, da $\$$ beide immer vereint, z. B. in Quetschpräparaten oder im Mehle, aufgefunden werden; sehr häufig säumt der hyaline Streifen die Fetzen der braunen Haut mit einem dicken Wulst ein (Fig. 6 bei y); er scheint aber auch etwas starr und brüchig zu sein, wodurch es geschieht, daß die gestreiften Zellen der braunen Schicht über die Bruchstellen des hyalinen Streifens hervorragen (Fig. 6 bei 2 und 3). An dem Streifen liegt eine dünnwandige Zellreihe (Fig. $4,3 a$ ), für welche der hyaline Streifen die Cuticula oder die cutinisierte Außenwand der Zellen darstellen dürfte.

Nebst der Epidermis sind die Bruchstücke der braunen Schicht mit dem damit fest verbundenen hyalinen Streifen die Leitelemente, die das Chenopodiummehl sicher und leicht erkennen lassen; auch der Nachweis des Chenopodium-Samens als Verunreinigung im Getreidemehl und zur Unterscheidung von den Wickensamenschalen ist unter Hinweis auf Fig. 3 sehr einfach.

Das Perisperm besteht als echtes Speichergewebe aus einem großzelligen, dünnwandigen Parenchym (Fig. 4, 5), das mit feinstkörniger Stärke dicht erfüllt ist. Mitunter sieht man rundliche Stärkekörper, die aus zahlreichen winzigen Stärkekörnchen zusammengesetzt sind. Einzelne Parenchymzellen sind durch besondere Größe ausgezeichnet.

Im Radicularteil schaltet sich zwischen der Samenschale und der Radicula noch eine Zellreihe ein, die der sog. Aleuronschicht zahlreicher Samen völlig gleicht (Fig. 2, 4; 5, 5; 7). Die Zellen sind im Querschnitt quadratisch, wo die Schicht einreihig ist, dagegen schmal rektangulär, wenn zwei Zellreihen vorkommen; in der Flächenansicht (Fig. 7) sind die Zellen polygonal, einzelne auffallend langgestreckt mit eingebuchteten Wänden; diese sind ziemlich stark und farblos. Der Inhalt ist insofern von dem der eigentlichen Aleuronzellen verschieden, als er nicht körnig, sondern als eine graugelbliche kompak t e Proteinmasse erscheint, die zusammenhängend aus der Zelle herausfällt und gewissermaßen einen Abguß des Zell-Lumens darstellt. Diese Schicht ist wohl ein Endospermrest.

Der $\mathrm{K}$ eim setzt sich aus den bekannten, mehr oder weniger kubischen, wie in eimem Mauerverband angeordneten Parenchymzellen zusammen, die reichlich Öl und winzige Proteinkörner enthalten. Da der Keim verhältnismäßig groß ist und als der Träger des Eiweißes und des Fettes fungiert, so ist die aus der chemischen Analyse sich ergebende aufällige Menge dieser Körper auch anatomisch erklärt.

Mit Rücksicht auf die chemische Zusammensetzung und unter Hinweis auf das uralte Nahrungsmittel Quinoa ist derChenopodiumsamen als Brotfrucht nicht gänzlich abzuweisen.

Vor einiger Zeit wurde ich von Prof. F. Netolitzky benachrichtigt, dab er im Chenopodiumsamen schwarze Partikel gefunden habe, die einigermaßen an Phytomelane erinnern. In der Tat sieht man im Keim, insbesondere im Würzelchen, schwarze Körnchen, deren Herkunft nicht klar ist. Die nähere Untersuchung über diese Körper steht noch aus. 
Erläuterungen zu den Abbildungen.

Alle Figuren betreffen den Samen von Chenopodium album $L$.

Fig. 1. Flächenschnitt durch den ganzen Samen. Vergr. 25. - rad Radicula (Würzelchen), kot Keimblätter, pe Keimnährgewebe (Perisperm).

Fig. 2. Quersehnitt durch den Samen längs der Linie $x-x$ von Fig. 1. Vergr. 60 - 1 Epidermis, 2 braune Sehicht (zweite Samenschalenschicht), $\neq$ Aleuronschicht, das übrige wie in Fig. 1.

Fig. 3. Epidermis der Samenschale von der Fläche. Vergr. 450.

Fig. 4. Partie eines Querschnittes durch den Samen in der Perispermgegend. Vergr. 450. -

1 Epidermis, 2 braune Zellschicht, 3 hyaliner Streifen, 3 a die dazugehörige Zellreihe,

5 Perispermgewebe.

Fig. 5. Partie eines Querschnittes durch den Samen in der Radiculargegend. Vergr. 400. Bezeichnungen wie in Fig. 4. $-2 a$ inmere Reiho der braunen Schicht. 4 Aleuronschicht.

Fig. 6. Die braune Schicht in der Fläche. Vergr. 400. -2 und 3 wie in Fig. 4. $-y$ walstartiger Rand von 3.

Fig. 7. Die Aleuronschicht 4 von Fig. 5 in der Fläche. Vergr. 450.

\section{Anhang: ,Das rote Gras66.}

Im Jahre 1903 wurde mir bekannt, daß in Rußland auch das sogenannte rote Gras als Brotfruchi Verwendung findet.

In der mir zugänglichen Literatur war eine so bezeichnete Pflanze nicht aufzufinden, und da ich der Meinung war, daß es sich um eine Graminee handle, so wandte ich mich an den bekannten Gramineenspezialisten Professor E. Hackel. Derselbe konnte ebenfalls keine Auskunft darüber geben, war aber so freundlich, bei dem Gramineenkenner Direktor Georg Westberg in Riga um Aufklärung anzufragen. Herr $W$ estberg konnte unter Vermittlung des Konservators des Botanischen Gartens in Petersburg, Hofrat Nikolaj Busch, nun folgende Auskunft geben (nach brieflicher Mitteilung vom $\frac{22}{4 .}$ April 1903).

Die Bezeichnung ,rotes Gras“ führen in verschiedenen Gebieten verschiedene Pflanzen, und zwar:

1. Amarantus paniculatus L. (im Gouv Voronez

2. Rumex acetosella L. (Gouv. Permj)

3. Trifolium medium L. (Gouv, Vladimir)

als Krasnaja trava bezeichnet.

4. Hypericum perforatum L. (Gouv. Minsk) als Krasnaja travica bezeichnet,

5. Angelica silvestris L. (Gouv. Podolisk) als Krasnoje zelje bezeichnet,

6. Atriplex rosea L. (im Süden) als Krasnaja trava bezeichnet.

Am wahrscheinlichsten wird nach Busch's Meinung Atriplex rosea oder Amarantus paniculatus dem Brot zugesetzt. - Trava heißt im Russischen "Gras" und "Kraut"; Kraut heißt auch noch zelje.

Dies der Bericht von Herrn Westberg. Wir sehen, daß als Brotfrucht ernstlich nur eine Meldenart, Atriplex, und eine Fuehsschwanzart, Amarantus, in Frage kommen können. Die Samen von Atriplex rosea sind wie die Chenopodiumsamen gebaut und ihre Verwendung zu Mebl erscheint daher ganz plausibel. Nach Heldreich diente die Pflanze in Griechenland zur Pottaschefabrikation; sie wurde 
nach Dragendorff auch als Antiscorbuticum und Antiscrophulosum gebraucht, die Sprosse wurden ähnlich wie Kappern genossen (Ascherson-Graebner, Synopsis $\mathrm{V}, 141)$. Als Brotfrucht ist die Pflanze nirgends bezeichnet.

Bezüglich Amarantus paniculatus scheint es sich nicht um die Linné'sche Art zu handeln, sondern un die von Hooker so benannte Pflanze, die von Linné Amarantus hypocondriacus, von Thellung (in Ascherson-Graebner, Synopsis V, 241) erythrostachys, roter Fuebschwanz genannt wurde. Eine Form desselben, A. hypochondriacus II., erythrostachys g. frumentarius Thellung, wird im nördlichen Ostindien als "Ranatampala" wegen der als "Landesi" bekannten Samen kultiviert. Die Samen sind schwarz. Eine weiß- oder hellsamige Abart (ochrospermus Thellung) steht wegen der zur Mehlbereitung verwendeten Samen in Tibet in Kultur.

Als Nutz- und Zierpflanze ist der rote Fuchsschwanz bekanntlich allgemein verbreitet. Da die Chenopodiaceen und die Amarantaceen miteinander sehr nabe verwandt sind, so weicht anch der Samenbau nicht wesentlich von dem des Chenopodium ab.

\section{Referate.}

\section{Allgemeine Bestandteile der Nahrungs- und Genußmittel.}

H. Rohonyi: Kolloid chemische Eiweißstudien. (Biochem. Zeitschr. 1913, 53, 179-209.) - Die Lösungen proteolytischer Enzyme besitzen die Eigenschaft, in gewissen Eiweib- und Albumoselösungen unter gewissen Bedingungen einen Niederschlag $z u$ erzeugen, der als eine komplexe Verbindung von Enzym und Eiweiß betrachtet wird. Es werden mehrere solche Reaktionen beschrieben und die Bedingungen ihres Zustandekommens ermittelt. Diese Reaktionen verlaufen auch nach Inalktivierung des Enzyms in der gleichen Weise. Die durch Leitfähigkeitsmessungen gewonnene Säurebindungskurve einer Eiweißlösung erfährt in ihrer Stetigkeit keine Unterbrechung, wenn inzwischen in der Lösung ein Eiweißniederschlag entsteht oder der gebildete Niederschlag gelöst wird. Die Säurebildung hängt also bloß von der absoluten Menge des Eiweißes ab, nicht aber von dessen Oberfläche. Vom Paranuclein konnte nachgewiesen werden, daß es kein hydrolytisches Produkt ist, sondern einen Casein-CaseoseKomplex darstellt. Von Robertso n's Paranuclein A weist Verf. nach, daf es eine komplexe Verbindung von einer Caseose und einem im Grübler'schen Pepsin enthaltenen eiweibartigen Körper ist. Endlich ist es nach den Versuchen des Verf.'s als wahrscheinlich anzusehen, daB die sog. "Plasteine" komplexe Albumose-EnzymVerbindungen darstellen.

Max Miiller.

A. Krzemecki : Über Jod-und Bromeinwirkung auf Proteinkörper. (Anz. Akad. Wiss. Krakau, Reihe H, 1911, 470 -488; Chem. Zentralbl. 1912, I, 2034-2036.) - $\mathrm{Zu}$ den Versuchen wurden koagulierte Eiweißkörper verwendet. In Methylalkohol nahmen die Proteinstoffe etwas mehr Jod auf als in Äther. Als Maximum für die Jod- und Bromaufnahmefäbigkeit wurden folgende Zahlen gefunden: für Eieralbumin $28,29-29,6 \% \mathrm{~J}$ und $18,05 \% \mathrm{Br}$, für Serumalbumin $28,48 \% \mathrm{~J}$ und $20,49 \% \mathrm{Br}$, für Casein $19,1-24,9 \% \mathrm{~J}$, für Pflanzeneiweiß $34,6 \% \mathrm{~J}$. Zur Feststellung der verschiedenartigen Bindung des $J$ und $\mathrm{Br}$ mit dem Eiweißmolekül wurden die jodierten Körper 1. der Einwirkung von konc. Essigsäure bei Siedetem- 